\title{
Perception of malaria and cultural diversity of antimalarial plants in three sympatric ethnic groups: Agni, Akyé and Gwa of Alépé Department (Southeast of Côte d'Ivoire)
}

Amadou Lamine Amadou DIOP ( $\square$ amadoulamine08@gmail.com )

Université Nangui Abrogoua (Côte d'Ivoire)

François Djah MALAN

Université Nangui Abrogoua

Distel Ménéké KOUGBO

Université Nangui Abrogoua

Research

Keywords: Malaria, Southeast Côte d'Ivoire, medicinal plants, Akan, Agni, Akyé, Gwa

Posted Date: January 6th, 2021

DOl: https://doi.org/10.21203/rs.3.rs-139062/v1

License: (c) (i) This work is licensed under a Creative Commons Attribution 4.0 International License.

Read Full License 


\section{Abstract \\ Background}

Ethnic groups have developed their own cultures expressed in the form of traditional health care systems. This study aimed to determine how three sympatric communities with different histories, perceive and manage malaria, a disease with a high prevalence rate in the region.

\section{Methods}

An ethnobotanical survey was carried out in 10 villages of Agni, Akyé and Gwa communities. Semistructured interviews were conducted with 290 informants within all three communities. A correspondence analysis associated to hierarchical clusters was used to determine the form of malaria shared within informants. Then, the free listing technique was performed to indicate the plant species which was most important for the respondents. Besides, the Venn diagram coupled to Jaccard similarity index was used to report the homogeneity on antimalarial plants species used within the three studied communities. Moreover, the Kruskal-Wallis test was used to compare the most common antimalarial plant within communities. Finally, the fidelity level index was used to identify the most preferred plant species used to cure various forms of malaria.

\section{Results}

The three ethnic groups have overall a similar perception of malaria with various symptoms. However, they did not use the same plants to cure one form of this disease. The study recorded 77 medicinal plants used to cure malaria, in which, a few such as Annickia polycarpa, Gymnanthemum amygdalinum, Alstonia boonei, Nauclea latifolia, Harungana madagascariensis, Ocimum gratissimum and Senna occidentalis were the most important. The analysis of intracultural knowledge on antimalarial plants revealed that informants have shared a high knowledge. Meanwhile, there is an intercultural convergence about common plants used within communities. Therefore, 20 antimalarial plant species were shared within communities and actively used, through time. Finally, in terms of antimalarial plants knowledge, Akyé and Gwa communities were closer than Agni communities.

\section{Conclusions}

In spite of their different histories, the close contact of communities promote the sharing of the knowledge. People use the same important plants to cure malaria and know five forms of malaria. Knowledge on antimalarial plants does not reach a stable climax, but could be evolved by trial and error, as effective cures malaria. 


\section{Introduction}

Medicinal plants are one of the most widely known values of traditional knowledge, as they provide primary health care [1]. Certainly, traditional medicine is an important source of health care in rural or tribal areas [2, 3]. In sub-Saharan countries such as Côte d'Ivoire [4, 5], Mali [6] and Guinea [7], people still use traditional medicine to cure many diseases as malaria.

Malaria is among the major vector-borne diseases that kill a lot of people in sub-Saharan Africa [8]. That disease constitutes a real public health issue and motivates the consultation and hospitalization in sanitary centres [9]. However, the raised costs of the sanitary cares lead many people in rural areas, to use traditional medicine as alternative for curing malaria [10]. Even so, in an intercultural region, a given ethnic group could know some species which may not be appreciated by another ethnic group or may even be ignored by them [11]. To that purpose, they do not use and value all plant species equally according to their needs in the same practice [12]. Those reasons could hide a variation in the perception of a particular disease and a different significance of plants for those communities [13], especially while they share the same geographical area with other communities [14].

In Africa, particularly in Côte d'Ivoire, numerous studies dealing with medicinal plant uses are simple lists of plants [15] or ethno-pharmacology uses against malaria $[4,16]$. Moreover, they were undertaken solely on intra-cultural background. Other studies in the same way, were undertaken for many diseases [17]. Comparative ethno-botanical studies among communities who share the same area are scarce. Nevertheless, such studies help to find which species are interchanged through communities and for which reasons $[1,18]$. Moreover, those studies analyse whether cultural diversity is reflected in the folk phytotherapy knowledge as shown by [11]. These authors argued, as noted by many others [1-18, 19] that the comparative study of medicinal plant knowledge among sympatric ethnic and/or local groups may be divided into two types. The first type focuses on traditional groups with comparatively long residence in the region and the second type concentrates on the comparison of medicinal plants use by ethnic groups with different times of residence in a given region. The present study is within the first statement. Indeed, in the Department of Alépé, in the southeastern region of Côte d'Ivoire, the Agni, Akyé and Gwa ethnic groups have lived in close proximity and contact for many centuries in the Department of Alépé. Agni and Akyé belong to the great Akan ethnic group [20-23], whereas Gwa ethnic groups were adopted by Akan $[24,25]$. Although close to the economic capital, Abidjan, this area is extremely difficult of access due to the poor state of the roads. Moreover, these communities are among those whose traditional practices are the least documented. Finally, these communities are in malaria high transmission zone with 200 to 300 confirmed cases per 1,000 inhabitants per year [26]. In the light of these backgrounds, the study aimed to (i) analyse the perception of different forms of malaria and (ii) to assess the importance of antimalarial plants species used among these ethnic groups. Moreover, this study aimed (iii) to determine the intracultural and the intercultural variations on antimalarial plant knowledge within ethnic groups.

\section{Material And Methods}




\section{Study area}

The Department of Alépé is located in Southeastern Côte d'Ivoire between $5^{\circ} 13^{\prime} 04.49^{\prime \prime}-5^{\circ} 55^{\prime} 22.06^{\prime \prime} \mathrm{N}$ and $3^{\circ} 25^{\prime} 25.25^{\prime \prime}-3^{\circ} 57^{\prime} 46.64^{\prime \prime} \mathrm{W}$ (Figure 1). The climate of this zone is equatorial and humid, characterized by four alternate seasons (two rainy seasons and two dry seasons). The annual rainfall is ranged from 1,200 to $1,600 \mathrm{~mm}$ and the annual temperature is $26.4^{\circ} \mathrm{C}$ with a variation of $3^{\circ} \mathrm{C}$. The vegetation type of the study area is a Guinean rainforest characterized by Eremospatha macrocarpa (Mann. \& Wendl.) Wendel. and Diospyros mannii Hiern [27].

The study area harbors three sympatric ethnic groups Agni, Akyé and Gwa. All of them are unequally spread within five sub-prefectures (Aboisso-Comoé, Alépé, Allosso, Danguira and Oghlwapo). These three ethnic groups have been settled in their present territory since the beginning of the 18th century, the Agni and the Akyé from present-day Ghana [22, 23], and the Gwa from Liberia [24, 25]. Upon arrival, these ethnic groups came into conflict for their current installation [28]. All three ethnic groups are essentially farmers.

\section{Ethnobotanical survey}

Several field trips were made in 10 villages of the study area from September 2017 to August 2019. A total of 290 informants were selected randomly with semi-structured interview [29]. At first step, free lists were collected through a house-to-house approach in each village. The seek items included forms of malaria, symptoms related to the forms of malaria, plants used for healing malaria.

Respondents were distributed as follows: 97 in Agni ethnic groups (40 men and 57 women), 97 in Akyé ethnic groups (35 men and 62 women) and finally 96 in Gwa ethnic groups (47 men and 49 women). For next step, walks in the surrounding bushes were organised with key informants in each village, to collect herbarium vouchers. At the final step, the lists collected during the first step was completed and herbarium vouchers identified at Nangui Abrogoua University.

\section{Data analysis}

A correspondence analysis (CA) combined to a hierarchical cluster analysis was performed in order to show the forms of malaria sharing between ethnic groups. The principle of this analysis is to establish the link between two sets of variables that constitute the rows and columns of a contingency table. This analysis was carried out with the frequency of quotation of the form of malaria by each ethnic group. That frequency of quotation was obtained to assess the extent of form in each ethnic group (Equation 1).

$$
F_{q}=\frac{n_{i}}{N} \times 100(1)
$$


Where $F_{q}$ is the frequency of quotation; $n$ is the number of times when the form of malaria was mentioned, and $\mathrm{N}$ is the total number of informants. When $\mathrm{F}_{\mathrm{q}} \geq 50 \%$ the form is considered as well known. Below this value, the form can be judged middle known $25 \leq \mathrm{F}_{\mathrm{q}} \leq 50 \%$ or low known $\mathrm{F}_{\mathrm{q}}<25 \%$.

Free listing technique was performed by Anthropac 4.0 following the Smith Index (Equation 2) in order to obtain the salience for each species [30].

$$
S=\left\{\sum[(L i-R j+1) / L j]\right\} / N(2)
$$

Where $S$ is the importance of quotations, $L i$ the length of a quotation list and $R j$ the rank of a quotation in the list and $N$ is the number of free lists (number of respondents). A high value of this index (close to 1 ) indicates the antimalarial plant species which is preferred and important for the respondents.

In addition, the Jaccard similarity Index [31] was used to analyse the homogeneity on antimalarial plants species and reports the similarity within the three studied communities (Equation 3 ).

$$
J I_{i j}=\frac{a}{a+b+c}
$$

$J /$ is the Jaccard similarity index, $a$ is the number of species common to any compared pair ethnic group $i$ and $j ; b$ is the number of species mentioned only by group $i$, and $c$ is the number of species mentioned solely by group $j$.

Then, the specific abundance shared by each pair of ethnic groups or exclusive to one ethnic group and the common species shared between the three ethnic groups were obtained with Venn diagram. This diagram shows the number of antimalarial plant species shared between ethnic groups. Moreover, a comparison test on the most common antimalarial plant species shared within the ethnic groups, were made with Kruskal-Wallis test. This test determines the intercultural convergence on knowledge exchanges between the ethnic groups.

Finally, the fidelity level (FL) index (Equation 4) was used to identify the preferred plants to heal various forms of malaria and shows the proportion of informants reporting the use of specific plants [32].

\section{$F L=N p / N \times 100 \%(4)$}

Where $N p$ is the number of respondents citing the use of species for a particular ailment and $N$ is the total number of respondents who cited the plants for any form. All statistical analyses were performed with $\mathrm{R}$ software (version 4.0.3).

\section{Results}


Knowledge and perception of malaria by the three ethnic groups

Six various terms are used to indicate six different forms of malaria according to each ethnic group (Table 1).

Table 1

Local names of malaria and their meanings.

\begin{tabular}{|llll|}
\hline $\begin{array}{l}\text { ethnic } \\
\text { group }\end{array}$ & $\begin{array}{l}\text { local } \\
\text { name }\end{array}$ & local perception & literal meaning \\
\hline Agni & ebunu & $\begin{array}{l}\text { ebunu fufue, ebunu kokole, ebunu } \\
\text { bile, ebunu ewengo, enwulo }\end{array}$ & $\begin{array}{l}\text { white malaria, yellow malaria, black } \\
\text { malaria, red malaria, birds malaria }\end{array}$ \\
\hline Akyé & shilo & $\begin{array}{l}\text { shilo fi, shilo nin, shilo bi, shilo poin, } \\
n^{\prime} \text { kaka, kpun shilo }\end{array}$ & $\begin{array}{l}\text { white malaria, yellow malaria, black } \\
\text { malaria, red malaria, birds malaria, } \\
\text { mystical malaria }\end{array}$ \\
\hline Gwa & djakoadjo & $\begin{array}{l}\text { djakoadjo popon, djakoadjo heni, } \\
\text { djakoadjo mlu, djakoadjo nuin, zoku, } \\
\text { djakoadjo montinin }\end{array}$ & $\begin{array}{l}\text { white malaria, yellow malaria, black } \\
\text { malaria, red malaria, birds malaria, } \\
\text { mystical malaria }\end{array}$ \\
\hline
\end{tabular}

Among these six different forms of malaria, five are expressed with symptoms. Meanwhile, the mystical malaria, another form appears without symptoms. Thus, the most common form is yellow malaria mentioned by $72.16 \%$ of informants, which means yellowish eyes, yellowish urine and fever. Then, white malaria identified by $58.76 \%$ means pale skin and edema. Besides, red malaria is indicated by $42.61 \%$ which means fever and reddish eyes. Moreover, black malaria is identified by $24.74 \%$, means fever and dark skin. Otherwise, $5.15 \%$ of informants have mentioned bird malaria which means disjointed movements, fever and pale skin. Finally, the mystical malaria is indicated by $1.37 \%$ of informants without symptoms. Table 2 summarizes the symptoms mentioned in the study. 
Table 2

Symptoms related to the forms of malaria.

\begin{tabular}{|c|c|c|c|c|c|c|}
\hline Symptom & $\begin{array}{l}\text { white } \\
\text { malaria } \\
(\%)\end{array}$ & $\begin{array}{l}\text { yellow } \\
\text { malaria (\%) }\end{array}$ & $\begin{array}{l}\text { black } \\
\text { malaria (\%) }\end{array}$ & $\begin{array}{l}\text { red } \\
\text { malaria } \\
(\%)\end{array}$ & $\begin{array}{l}\text { bird } \\
\text { malaria } \\
(\%)\end{array}$ & $\begin{array}{l}\text { mystical } \\
\text { malaria }\end{array}$ \\
\hline ache/tiredness & 4.73 & 6.91 & 6.29 & 9.17 & & \\
\hline $\begin{array}{l}\text { anemia/dark } \\
\text { skin }\end{array}$ & 2.21 & 0.43 & 9.09 & 3.98 & & \\
\hline cold sore & 7.57 & 3.24 & & 0.92 & & \\
\hline constipation & 0.95 & 1.3 & 6.29 & 5.81 & & \\
\hline $\begin{array}{l}\text { disjointed } \\
\text { movements }\end{array}$ & & & & & 29.73 & \\
\hline distension & 5.36 & 0.86 & 0.7 & & 5.41 & \\
\hline dizziness & 2.52 & 0.43 & 2.1 & 0.61 & & \\
\hline edema & 15.46 & & & 0.61 & & \\
\hline headache & 2.84 & 6.48 & 2.1 & 1.22 & & \\
\hline hot body/fever & 9.15 & 15.98 & 11.19 & 11.32 & 27.03 & \\
\hline insomnia & 2.21 & 2.16 & 7.69 & 1.84 & & \\
\hline $\begin{array}{l}\text { lack/loss of } \\
\text { appetite }\end{array}$ & 2.84 & 5.83 & 6.29 & 6.12 & & \\
\hline madness & 2.84 & 1.08 & 4.9 & 1.53 & & \\
\hline pale skin & 24.61 & 6.26 & 7.69 & 9.79 & 27.03 & \\
\hline reddish eyes & & 0.65 & 6.29 & 10.4 & & \\
\hline reddish urine & 0.63 & 0.65 & 7.69 & 9.17 & & \\
\hline sleepiness & 0.32 & & 2.1 & 2.45 & & \\
\hline $\begin{array}{l}\text { stomach } \\
\text { wound }\end{array}$ & 1.26 & 2.81 & 4.9 & 4.59 & & \\
\hline vomiting & 2.21 & 1.73 & 1.4 & 5.2 & & \\
\hline yellowish eyes & 4.1 & 21.81 & 6.99 & 7.34 & & \\
\hline yellowish urine & 8.20 & 21.38 & 6.29 & 7.95 & 10.81 & \\
\hline
\end{tabular}

The free lists on the form show that each ethnic group has mentioned two forms of malaria on average, in spite of the various forms of malaria (Fig. 2). 
The distribution of the forms of malaria within the ethnic groups is divided into two groups, according to the frequency of quotation (Table 3).

Table 3

Most common forms of malaria according to ethnic groups.

\begin{tabular}{|llll|}
\hline form of malaria & $\mathrm{F}_{\mathrm{qAgni}}(\%)$ & $\mathrm{F}_{\mathrm{qAkyé}}(\%)$ & $\mathrm{F}_{\mathrm{qGwa}}(\%)$ \\
\hline "white"malaria & 62.26 & 46.59 & 65.98 \\
\hline "yellow" malaria & 88.68 & 87.5 & 40.21 \\
\hline "black" malaria & 24.53 & 20.45 & 28.87 \\
\hline "bird" malaria & 02.83 & 06.82 & 06.19 \\
\hline "red" malaria & 36.79 & 15.91 & 07.32 \\
\hline "mystical" malaria & & 03.41 & 01.03 \\
\hline
\end{tabular}

The first group (G1) included the forms mentioned by Gwa ethnic groups. Meanwhile, the second group (G2) is formed by the forms mentioned by both Agni and Akyé ethnic groups (Fig. 3).

The distribution on the form of malaria was not significantly different (Chi squared $=0.6874296$; $p$-value $=0.9999699$ ). Agni and Akyé ethnic groups are closer on forms of malaria than Gwa ethnic groups (Fig. 4).

Diversity and intercultural relations of antimalarial plant species

Seventy-seven (77) species, distributed in 71 genera and 38 families were collected (Table 4). The most represented families were Lamiaceae, Asteraceae and Leguminosae with five species. They were mainly composed of 67 trees and shrubs, 10 herbaceous plants and one liana. 
Table 4

Cultural importance of antimalarial plant species used in the study area.

\begin{tabular}{|c|c|c|c|c|c|c|c|c|}
\hline & & & Agni & & Akyé & & Gwa & \\
\hline Family & Species & Voucher\# & $\begin{array}{l}\mathrm{Fq} \\
(\%)\end{array}$ & S & $\begin{array}{l}\mathrm{Fq} \\
(\%)\end{array}$ & $S$ & $\begin{array}{l}\mathrm{Fq} \\
(\%)\end{array}$ & $S$ \\
\hline \multirow[t]{2}{*}{ Acanthaceae } & $\begin{array}{l}\text { Justicia tenella } \\
\text { (Nees) T.Anderson }\end{array}$ & DL003 & & & & & 1.04 & 0.01 \\
\hline & $\begin{array}{l}\text { Phaulopsis ciliata } \\
\text { (Wild.) Hepper }\end{array}$ & DL087 & & & 2.08 & 0.02 & & \\
\hline Alliaceae & Allium sativum L. & DL055 & 1.03 & 0.01 & & & & \\
\hline \multirow[t]{3}{*}{ Anacardiaceae } & $\begin{array}{l}\text { Mangifera indica } \\
\text { L. }\end{array}$ & DL021 & 1.03 & 0.01 & 6.25 & 0.03 & 3.13 & 0.02 \\
\hline & $\begin{array}{l}\text { Spondias mombin } \\
\text { L. }\end{array}$ & DL011 & & & 2.08 & 0.02 & 4.17 & 0.04 \\
\hline & $\begin{array}{l}\text { Trichoscypha } \\
\text { arborea (A.Chev.) } \\
\text { A.Chev. }\end{array}$ & DL093 & & & 6.25 & 0.05 & 1.04 & 0.01 \\
\hline \multirow[t]{3}{*}{ Annonaceae } & $\begin{array}{l}\text { Annickia } \\
\text { polycarpa (DC.) } \\
\text { Setten \& Maas }\end{array}$ & DL083 & 44.33 & 0.23 & 49.48 & 0.35 & 28.13 & 0.19 \\
\hline & $\begin{array}{l}\text { Monodora } \\
\text { myristica } \\
\text { (Gaertn.) Dunal }\end{array}$ & DL123 & & & & & 1.04 & 0.00 \\
\hline & $\begin{array}{l}\text { Xylopia } \\
\text { aethiopica } \\
\text { (Dunal) A.Rich. }\end{array}$ & DL175 & & & 1.04 & 0.01 & 1.04 & 0.00 \\
\hline \multirow[t]{4}{*}{ Apocynaceae } & $\begin{array}{l}\text { Alstonia boonei } \\
\text { De Wild. }\end{array}$ & DL067 & 38.14 & 0.27 & 35.05 & 0.26 & 17.71 & 0.10 \\
\hline & $\begin{array}{l}\text { Hunteria } \\
\text { umbellata (K. } \\
\text { Schum.) Hallier f. }\end{array}$ & DL070 & 2.06 & 0.01 & & & & \\
\hline & $\begin{array}{l}\text { Picralima nitida } \\
\text { (Stapf) T.Durand } \\
\text { \& H. Durand }\end{array}$ & DL089 & & & 1.03 & 0.05 & 1.04 & 0.00 \\
\hline & $\begin{array}{l}\text { Rauvolfia } \\
\text { vomitoria Afzel. }\end{array}$ & DL075 & 14.43 & 0.09 & 10.42 & 0.09 & 7.29 & 0.05 \\
\hline Arecaceae & Cocos nucifera L. & & & & & & 7.29 & 0.03 \\
\hline Asteraceae & $\begin{array}{l}\text { Ageratum } \\
\text { conyzoides } \mathrm{L} .\end{array}$ & DL002 & & & & & 11.46 & 0.01 \\
\hline
\end{tabular}




\begin{tabular}{|c|c|c|c|c|c|c|c|c|}
\hline & & & Agni & & Akyé & & Gwa & \\
\hline & $\begin{array}{l}\text { Chromolaena } \\
\text { odorata (L.) } \\
\text { R.M.King \& H. } \\
\text { Rob. }\end{array}$ & DL057 & 5.21 & 0.05 & 1.04 & 0.01 & & \\
\hline & $\begin{array}{l}\text { Gymnanthemum } \\
\text { amygdalinum } \\
\text { (Delile) Sch.Bip. } \\
\text { ex Walp. }\end{array}$ & DL013 & 26.80 & 0.16 & 19.79 & 0.11 & 35.42 & 0.25 \\
\hline & $\begin{array}{l}\text { Microglossa } \\
\text { pyrifolia (Lam.) } \\
\text { Kuntze }\end{array}$ & DL018 & & & & & 1.04 & 0.01 \\
\hline & $\begin{array}{l}\text { Struchium } \\
\text { sparganophorum } \\
\text { (L.) Kuntze }\end{array}$ & DL038 & & & & & 4.17 & 0.02 \\
\hline Bignoniaceae & $\begin{array}{l}\text { Newbouldia laevis } \\
\text { (P.Beauv.) Seem. } \\
\text { ex Bureau }\end{array}$ & DL094 & & & & & 2.08 & 0.02 \\
\hline Bromeliaceae & $\begin{array}{l}\text { Ananas comosus } \\
\text { (L.) Merr. }\end{array}$ & & & & 1.04 & 0.00 & 2.08 & 0.01 \\
\hline Cannabaceae & $\begin{array}{l}\text { Trema orientalis } \\
\text { (L.) Blume }\end{array}$ & DL034 & 2.06 & 0.01 & 2.06 & 0.01 & & \\
\hline Cannaceae & Canna indica L. & DL054 & 10.31 & 0.07 & & & & \\
\hline Caricaceae & Carica papaya L. & DL056 & 18.56 & 0.09 & 14.43 & 0.08 & 19.79 & 0.08 \\
\hline \multirow[t]{3}{*}{ Combretaceae } & $\begin{array}{l}\text { Terminalia } \\
\text { catappa L. }\end{array}$ & DL076 & 3.09 & 0.01 & & & 1.04 & 0.01 \\
\hline & $\begin{array}{l}\text { Terminalia } \\
\text { ivorensis A.Chev. }\end{array}$ & DL078 & & & 2.08 & 0.02 & & \\
\hline & $\begin{array}{l}\text { Terminalia } \\
\text { superba Engl. \& } \\
\text { Diels }\end{array}$ & DL077 & 1.03 & 0.01 & & & & \\
\hline Crassulaceae & $\begin{array}{l}\text { Kalanchoe } \\
\text { crenata (Andrews) } \\
\text { Haw. }\end{array}$ & DL086 & & & 1.04 & 0.01 & & \\
\hline Cucurbitaceae & $\begin{array}{l}\text { Momordica } \\
\text { charantia L. }\end{array}$ & DL071 & 7.22 & 0.05 & 9.38 & 0.07 & 25 & 0.13 \\
\hline Ebenaceae & $\begin{array}{l}\text { Diospyros sanza- } \\
\text { minika A.Chev. }\end{array}$ & DL112 & & & & & 1.04 & 0.01 \\
\hline
\end{tabular}

$F_{q}$ : Frequency of quotation of plant species; $S$ : importance of quotation of plant species 


\begin{tabular}{|c|c|c|c|c|c|c|c|c|}
\hline & & & Agni & & Akyé & & Gwa & \\
\hline \multirow[t]{4}{*}{ Euphorbiaceae } & $\begin{array}{l}\text { Alchornea } \\
\text { cordifolia } \\
\text { (Schumach \& } \\
\text { Thonn.) Müll.Arg. }\end{array}$ & DL007 & 15.46 & 0.06 & 13.54 & 0.07 & 11.46 & 0.07 \\
\hline & $\begin{array}{l}\text { Macaranga barteri } \\
\text { Müll.Arg. }\end{array}$ & DL183 & & & 1.03 & 0.01 & & \\
\hline & $\begin{array}{l}\text { Manihot } \\
\text { esculenta Crantz }\end{array}$ & DL061 & 6.19 & 0.05 & 6.19 & 0.04 & & \\
\hline & $\begin{array}{l}\text { Ricinodendron } \\
\text { heudelotii (Baill.) } \\
\text { Pierre ex Heckel }\end{array}$ & DL095 & & & & & 1.04 & 0.00 \\
\hline \multirow[t]{2}{*}{ Hypericaceae } & $\begin{array}{l}\text { Harungana } \\
\text { madagascariensis } \\
\text { Lam. ex Poir. }\end{array}$ & DL048 & 28.87 & 0.17 & 14.43 & 0.10 & 6.25 & 0.05 \\
\hline & $\begin{array}{l}\text { Vismia guineensis } \\
\text { (L.) Choisy }\end{array}$ & DL079 & 6.19 & 0.04 & & & & \\
\hline Irvingiaceae & $\begin{array}{l}\text { Irvingia } \\
\text { gabonensis } \\
\text { (AubryLecomte ex } \\
\text { O'Rorke) Baill. }\end{array}$ & DL085 & & & 3.09 & 0.02 & & \\
\hline \multirow[t]{5}{*}{ Lamiaceae } & $\begin{array}{l}\text { Hoslundia } \\
\text { opposita Vahl. }\end{array}$ & DL010 & 11.34 & 0.06 & 2.06 & 0.02 & 3.13 & 0.02 \\
\hline & $\begin{array}{l}\text { Ocimum } \\
\text { gratissimum L. }\end{array}$ & DL005 & 22.68 & 0.15 & 11.34 & 0.05 & 14.58 & 0.09 \\
\hline & $\begin{array}{l}\text { Plectranthus } \\
\text { monostachyus } \\
\text { (P.Beauv.) } \\
\text { B.J.Pollard }\end{array}$ & DL001 & 1.03 & 0.00 & & & 1.04 & 0.01 \\
\hline & $\begin{array}{l}\text { Tectona grandis } \\
\text { L.f. }\end{array}$ & DL064 & 11.34 & 0.05 & 1.03 & 0.01 & 3.13 & 0.02 \\
\hline & $\begin{array}{l}\text { Vitex grandifolia } \\
\text { Gürke }\end{array}$ & DL148 & & & 1.03 & 0.01 & & \\
\hline \multirow[t]{2}{*}{ Leguminosae } & $\begin{array}{l}\text { Distemonanthus } \\
\text { benthamianus } \\
\text { Baill. }\end{array}$ & DL069 & 2.06 & 0.01 & 5.15 & 0.03 & & \\
\hline & $\begin{array}{l}\text { Parkia bicolor } \\
\text { A.Chev. }\end{array}$ & DL147 & 4.12 & 0.02 & & & & \\
\hline
\end{tabular}

$F_{\mathrm{q}}$ : Frequency of quotation of plant species; $\mathrm{S}$ : importance of quotation of plant species 


\begin{tabular}{|c|c|c|c|c|c|c|c|c|}
\hline & & & Agni & & Akyé & & Gwa & \\
\hline & $\begin{array}{l}\text { Piliostigma } \\
\text { thonningii } \\
\text { (Schumach.) } \\
\text { Milne-Redh. }\end{array}$ & DL090 & & & 1.03 & 0.01 & & \\
\hline & $\begin{array}{l}\text { Senna alata (L.) } \\
\text { Roxb. }\end{array}$ & DL063 & & & & & 4.17 & 0.04 \\
\hline & $\begin{array}{l}\text { Senna } \\
\text { occidentalis (L.) } \\
\text { Link }\end{array}$ & DL023 & 21.65 & 0.15 & 16.49 & 0.11 & 20.83 & 0.13 \\
\hline Loganiaceae & $\begin{array}{l}\text { Anthocleista } \\
\text { nobilis G.Don }\end{array}$ & DL068 & 3.09 & 0.02 & 7.22 & 0.06 & & \\
\hline \multirow[t]{4}{*}{ Malvaceae } & $\begin{array}{l}\text { Cola nitida (Vent.) } \\
\text { Schott \& Endl. }\end{array}$ & DL096 & & & & & 4.17 & 0.03 \\
\hline & $\begin{array}{l}\text { Sida acuta Burm. } \\
\text { f. }\end{array}$ & DL091 & & & 3.09 & 0.01 & & \\
\hline & $\begin{array}{l}\text { Tarrietia utilis } \\
\text { (Sprague) } \\
\text { Sprague }\end{array}$ & DL092 & & & 3.09 & 0.03 & & \\
\hline & $\begin{array}{l}\text { Theobroma cacao } \\
\text { L. }\end{array}$ & DL065 & 2.06 & 0.01 & & & & \\
\hline \multirow[t]{2}{*}{ Meliaceae } & $\begin{array}{l}\text { Azadirachta } \\
\text { indica A.Juss. }\end{array}$ & DL097 & & & & & 8.33 & 0.07 \\
\hline & $\begin{array}{l}\text { Khaya ivorensis } \\
\text { A.Chev. }\end{array}$ & DL098 & & & 15.46 & 0.10 & 4.17 & 0.03 \\
\hline Moringaceae & $\begin{array}{l}\text { Moringa oleifera } \\
\text { Lam. }\end{array}$ & DL099 & & & & & 2.08 & 0.00 \\
\hline Musaceae & $\begin{array}{l}\text { Musa paradisiaca } \\
\text { L. }\end{array}$ & DL062 & 15.46 & 0.09 & 14.43 & 0.07 & 5.21 & 0.02 \\
\hline Myrtaceae & $\begin{array}{l}\text { Psidium guajava } \\
\text { L. }\end{array}$ & DL082 & & & 3.09 & 0.03 & 1.04 & 0.01 \\
\hline Ochnaceae & $\begin{array}{l}\text { Lophira alata } \\
\text { Banks ex } \\
\text { C.F.Gaertn. }\end{array}$ & DL032 & 17.53 & 0.11 & 10.31 & 0.04 & 2.08 & 0.01 \\
\hline Pandaceae & $\begin{array}{l}\text { Microdesmis } \\
\text { keayana } \\
\text { J.Léonard }\end{array}$ & DL035 & 1.03 & 0.01 & & & 4.17 & 0.01 \\
\hline
\end{tabular}

$F_{\mathrm{q}}$ : Frequency of quotation of plant species; $\mathrm{S}$ : importance of quotation of plant species 


\begin{tabular}{|c|c|c|c|c|c|c|c|c|}
\hline & & & Agni & & Akyé & & Gwa & \\
\hline Piperaceae & $\begin{array}{l}\text { Piper guineense } \\
\text { Schumach. \& } \\
\text { Thonn. }\end{array}$ & DL028 & 1.03 & 0.01 & & & & \\
\hline \multirow[t]{3}{*}{ Poaceae } & $\begin{array}{l}\text { Bambusa vulgaris } \\
\text { Schrad. ex J.C. } \\
\text { Wendl. }\end{array}$ & DL044 & 5.15 & 0.03 & & & 14.58 & 0.11 \\
\hline & $\begin{array}{l}\text { Cymbopogon } \\
\text { citratus (DC.) } \\
\text { Stapf }\end{array}$ & DL060 & 2.06 & 0.02 & 1.03 & 0.01 & 1.04 & 0.01 \\
\hline & $\begin{array}{l}\text { Saccharum } \\
\text { officinarum L. }\end{array}$ & DL100 & & & & & 2.08 & 0.02 \\
\hline Rhizophoraceae & $\begin{array}{l}\text { Anopyxis } \\
\text { klaineana (Pierre) } \\
\text { Engl. }\end{array}$ & DL081 & & & & & 1.04 & 0.00 \\
\hline \multirow[t]{3}{*}{ Rubiaceae } & $\begin{array}{l}\text { Mitragyna } \\
\text { ledermannii } \\
\text { (K.Krause) } \\
\text { Ridsdale }\end{array}$ & DL027 & 18.56 & 0.1 & 16.49 & 0.10 & 13.54 & 0.10 \\
\hline & $\begin{array}{l}\text { Nauclea } \\
\text { diderrichii (De } \\
\text { Wild. \& T.Durand) } \\
\text { Merr. }\end{array}$ & DL074 & 22.68 & 0.11 & & & 1.04 & 0.01 \\
\hline & $\begin{array}{l}\text { Nauclea latifolia } \\
\text { Sm. }\end{array}$ & DL033 & 21.65 & 0.16 & 3.09 & 0.02 & 32.29 & 0.27 \\
\hline \multirow[t]{3}{*}{ Rutaceae } & $\begin{array}{l}\text { Citrus } \\
\text { aurantiifolia } \\
\text { (Christm.) Swingle }\end{array}$ & DL058 & 5.15 & 0.04 & 3.09 & 0.02 & 18.75 & 0.09 \\
\hline & $\begin{array}{l}\text { Citrus aurantium } \\
\text { L. }\end{array}$ & DL059 & 8.25 & 0.07 & 4.12 & 0.01 & 1.04 & 0.01 \\
\hline & $\begin{array}{l}\text { Zanthoxylum } \\
\text { gilletii (De Wild.) } \\
\text { P.G.Waterman }\end{array}$ & DL080 & 1.03 & 0.01 & & & & \\
\hline \multirow[t]{2}{*}{ Sapindaceae } & $\begin{array}{l}\text { Blighia unijugata } \\
\text { Baker }\end{array}$ & DL084 & & & 3.09 & 0.02 & & \\
\hline & $\begin{array}{l}\text { Paullinia pinnata } \\
\text { L. }\end{array}$ & DL029 & 4.12 & 0.04 & & & & \\
\hline Solanaceae & $\begin{array}{l}\text { Physalis angulata } \\
\text { L. }\end{array}$ & DL088 & & & 1.03 & 0.01 & & \\
\hline
\end{tabular}

$\mathrm{F}_{\mathrm{q}}$ : Frequency of quotation of plant species; $\mathrm{S}$ : importance of quotation of plant species 


\begin{tabular}{|c|c|c|c|c|c|c|c|c|}
\hline & & & Agni & & Akyé & & Gwa & \\
\hline & $\begin{array}{l}\text { Solanum nigrum } \\
\text { L. }\end{array}$ & DL039 & & & 3.09 & 0.02 & & \\
\hline \multirow[t]{2}{*}{ Urticaceae } & $\begin{array}{l}\text { Musanga } \\
\text { cecropioides R.Br. }\end{array}$ & DL072 & 3.09 & 0.02 & & & 6.25 & 0.03 \\
\hline & $\begin{array}{l}\text { Myrianthus } \\
\text { arboreus P.Beauv. }\end{array}$ & DL073 & 4.12 & 0.03 & & & & \\
\hline Zingiberaceae & $\begin{array}{l}\text { Aframomum } \\
\text { melegueta } \\
\text { (Roscoe) } \\
\text { K.Schum. }\end{array}$ & DL066 & 1.03 & 0.01 & & & & \\
\hline
\end{tabular}

Among the 77 collected species, 20 plant species (25.97\%) were shared within all ethnic groups. While 12 plant species were exclusive to Agni and Akyé and 15 to Gwa people (Fig. 5). Nevertheless, the KruskalWallis test reveals that there is no significant difference (Chi squared $=3.266 ; \mathrm{df}=2 ; \mathrm{p}$-value $=0.1953$ ), within all ethnic groups on common plants species.

(Table 4 must be here)

All values are below 0.5 i.e $50 \%$ which means that there is a low knowledge sharing of antimalarial plants species (Table 5). Thus, Akyé are closer to Gwa ethnic groups whereas, the knowledge of Agni ethnic groups is specifics of them.

Table 5

Similarity (\%) on antimalarial plant species

used among ethnic groups.

\begin{tabular}{|ccc|}
\hline & Agni & Akyé \\
\hline Akyé & 40.3 & \\
\hline Gwa & 40.00 & 41.5 \\
\hline
\end{tabular}

Importance of antimalarial plant species within each ethnic group

Overall from 77 plants used against malaria, only seven (09.09\%) are culturally important (with $S \geq 0.15$ for threshold). Their values change from one ethnic group to another (Table 4). Thus, the most important species were Annickia polycarpa (DC). Setten \& Maas $\left[\left(S_{\text {Akyé }}=0.35 ; F_{\text {qAkyé }}=49.48 \%\right.\right.$, Fig. 6a); $\left(S_{\text {Agni }}=0.23\right.$; $\left.\left.\mathrm{F}_{\mathrm{qAgni}}=44.33 \%\right) ;\left(\mathrm{S}_{\mathrm{Gwa}}=0.19 ; \mathrm{F}_{\mathrm{qGwa}}=28.13 \%\right)\right]$, Gymnanthemum amygdalinum (Delile) Sch. Bip. ex Walp. $\left[\left(\mathrm{S}_{\mathrm{Gwa}}=0.25 ; \mathrm{F}_{\mathrm{qGwa}}=35.42 \%\right) ;\left(\mathrm{S}_{\mathrm{Agni}}=0.16 ; \mathrm{F}_{\mathrm{qAgni}}=26.80 \%\right)\right]$, Alstonia boonei De Wild. [( $\mathrm{S}_{\mathrm{Agni}}=0.27$; $\mathrm{Fq}_{\mathrm{Agni}}=38.14 \%$, Fig. 6b); $\left.\left(\mathrm{S}_{\mathrm{Akyé}}=0.26 ; \mathrm{F}_{\mathrm{qAkyé}}=35.05 \%\right)\right]$, Nauclea latifolia $\mathrm{Sm}$. $\left(\mathrm{S}_{\mathrm{Gwa}}=0.27 ; \mathrm{F}_{\mathrm{qGwa}}=32.29 \%\right)$; 
Harungana madagascariensis Lam. ex Poir. $\left(\mathrm{S}_{\mathrm{Agni}}=0.17 ; \mathrm{F}_{\mathrm{qAgni}}=28.87 \%\right)$; Ocimum gratissimum $\mathrm{L}$.

$\left(S_{\text {Agni }}=0.15 ; F_{q A g n i}=22.68 \%\right)$ and Senna occidentalis $(L$. $)$ Link $\left(S_{\text {Agni }}=0.15 ; F_{q A g n i}=21.65 \%\right)$. Figure 6 shows two plant species most important in the study area.

The fidelity level (FL) of the recorded plants was less than $40 \%$ suggesting that none of them are really specific to a particular form of malaria. Thus, the plants of high cultural values mentioned above are frequently used to cure various forms of malaria (Table 6).

Table 6

Fidelity level of most common antimalarial plants of Agni, Akyé and Gwa people.

\begin{tabular}{|c|c|c|c|c|c|}
\hline & FL (\%) & & & & \\
\hline Species & $\begin{array}{l}\text { white } \\
\text { malaria }\end{array}$ & $\begin{array}{l}\text { yellow } \\
\text { malaria }\end{array}$ & $\begin{array}{l}\text { black } \\
\text { malaria }\end{array}$ & $\begin{array}{l}\text { bird } \\
\text { malaria }\end{array}$ & $\begin{array}{l}\text { red } \\
\text { malaria }\end{array}$ \\
\hline Alstonia boonei De Wild. & 30.14 & 30.82 & 26.42 & & 23.89 \\
\hline Annickia polycarpa (DC.) Setten \& Maas & 30.82 & 38.99 & 22.64 & & 29.2 \\
\hline Carica papaya L. & 23.29 & 16.98 & 26.42 & & \\
\hline Distemonanthus benthamianus Baill. & & & & 37.5 & \\
\hline $\begin{array}{l}\text { Gymnanthemum amygdalinum (Delile) } \\
\text { Sch. Bip. ex Walp. }\end{array}$ & 36.3 & 21.38 & 32.08 & 25 & 30.97 \\
\hline Momordica charantia L. & 19.86 & 10.69 & 22.64 & & 12.39 \\
\hline Musa paradisiaca $\mathrm{L}$. & & & & 37.5 & \\
\hline Nauclea latifolia Sm. & & 18.87 & & & 22.12 \\
\hline
\end{tabular}

The intracultural analysis of antimalarial plants lists suggested that knowledge is widely shared in each community (Fig. 7) as the level of saturation of the respondents was quickly reached: after the 15th respondent out of 97 in Agni, the 19th out of 97 in Akyé and the 17th out of 96 informants in Gwa ethnic groups.

However, the Gwa recorded the longest lists (average $=4$ plants), followed by the Agni (average $=4$ plants), and then the Akyé with an average of three plants (Fig. 8).

\section{Discussion}

This study aimed to analyse the perception of various forms of malaria in three sympatric ethnic groups with various sociolinguistic backgrounds. Different terms were used to indicate six forms of malaria across ethnic groups. This diversity on the forms of malaria is not unique to our study area. In fact, in Africa several studies have shown various communities whose have distinguished many forms of 
malaria. For example, in Mali the communities of Sélingué subdistricts, have identified malaria through five forms sumaya, nènèdimi, djontè, djokoadjo and farigan [6]. Besides, in Zimbabwe, in the Chipinge District, traditional healers have recognized two forms of malaria, muswarara and ndangaranga [10]. However, the knowledge on the symptoms changes from one ethnic group to another. In fact, this variation of the symptoms was allowed to distinguish a simple form and a serious form of malaria. Thus, the symptoms as disjointed movements, fever, pale skin have been frequently reported by the ethnic groups in our study area. In the similar observations, the communities of Chipinge in Zimbabwe have mentioned those symptoms [10]. Moreover, in Mali the Sélingué communities have considered convulsions as an uncomplicated symptom of malaria [6]. Traditional medicine distinguishes a varied range of symptoms. Those symptoms evolve from one country to another. In fact, the variations of symptoms depend on traditional practices and cultural backgrounds [33,34]. Better, those variations depend on the accessibility of rural communities to conventional medicine [10]. Therefore, local perceptions of malaria can be matched to conventional medicine. In fact, conventional medicine distinguishes two main forms of malaria (simple and complicated form) with many variations [35]. However, traditional medicine as well as conventional medicine cannot cope some disorders as mystical malaria and cerebral malaria. Those difficulties have conducted to the integration of traditional medicine in the region health system $[6-10,33]$.

However, the high transmission of malaria creates a tendency for people to catch that disease. Indeed, the susceptibility to the malaria changes from one country to another according to cultural backgrounds and was revealed by the ethnic groups behavior. In fact, the immunogenetic factors might be taken in account for distinguishing the susceptibility to malaria [34]. For example, in Mali two sympatric communities have been compared. Their studies have shown that in spite of fever which is a common symptom of malaria, Peulh communities are more susceptible to catch malaria than Dogon communities.

Traditional medicine is a part of people's culture and is closely linked to their beliefs. In fact, people combine religion, sorcery and interpersonal conflict into a single form of belief and practice [36]. As an example, Amazonian people of Upper Rio Negro of Brazil associated malaria to spiritual beings and used to be cured with the blessings of shamans [37].

However, the other issue raised in this study is the importance of antimalarial plants species. As well as mentioned by some authors [37,38], the use of plants depends on the culture. Indeed, the doctrine of signatures [39], has been used as a means to understand the medicinal plant selection process in traditional cultures. Moreover, for some authors [40] in Mexico and in Côte d'Ivoire [17], the organoleptic properties (bitter taste of bark stems and leaves or yellow color of the bark stems and the shape of plant organs) indicate that a given plant, has a medicinal potential and a therapeutic application. Thus, several studies have confirmed the antiplasmodial activities of the most important antimalarial plant species mentioned in this study. For example, stem bark of Annickia polycarpa [41], stem bark of Nauclea latifolia [42], stem bark of Harungana madagascariensis [43], leaves of Gymnanthemum amygdalinum [44], leaves of Ocimum gratissimum [45] and leaves of Senna occidentalis [46] were confirmed efficiency to manage malaria and related symptoms. However, the stem bark of Alstonia boonei was revealed inactive 
for inhibition concentration higher than $50 \mu \mathrm{g} / \mathrm{ml}$ against Plasmodium falciparum in, in vitro culture [43]. Even so, this plant species was prescribed against shiver and aches [15] in both Côte d'Ivoire and Ghana. For example, in Ghana, the leaves of Alstonia boonei have shown that the alkaloid extract of the species have an anti-plasmodial activity at $8.4 \mu \mathrm{g} / \mathrm{ml}$ [47]. Thus, the efficiency of a given plants depends on the parts of plant which were used differently by people to treat malaria [48].

Although the most important antimalarial plants above, have already been mentioned in literature for their antiplasmodial activities, there is no reference in the literature describing some antiplasmodial activities about Blighia unijugata Baker, Diospyros sanzaminika A.Chev., Cola nitida (Vent.) Schott \& Endl., Macaranga barteri Müll.Arg., Parkia bicolor A.Chev., Plectranthus monostachyus (P.Beauv.) B.J.Pollard, Tarrietia utilis (Sprague) Sprague and Vitex grandifolia Gürke. These plant species were less known in our study. Nevertheless, these plant species are known for their analgesic, feverishness and antianaemic properties [15], which represent remedies against certain symptoms of malaria. There is a lack of information exchanges about their uses among informants $[3,9]$.

On the other hand, the similarity rate between antimalarial plant species was under $50 \%$. The geographical proximity of ethnic groups have influenced the local culture in the uses of medicinal plants $[39,49]$. For example, in Pakistan, the communities of Dhirkot, Azad Jammu and Kashmir which share the same vegetation, have the same knowledge on the medicinal plants [3]. In the similar case, the communities of Allada in Benin have shared their knowledge [9].

The different cultural backgrounds have explained, however the uses of plant species to cure malaria. In fact, in our study the greatest amount of plant species was recorded in the family of Lamiaceae, Asteraceae, Leguminosae and Apocynaceae, whereas, in Abidjan plant species belonging to Rubiaceae, Combreataceae, Leguminosae and Meliaceae families were mainly used for their antimalarial properties. Therefore, trees and shrubs were more used than herbaceous plants. Inversely, in Brazil herbaceous plants were observed by [50].

The last issue of this study is to determine the intracultural and intercultural variations of the knowledge within ethnic groups. Thus, the local knowledge on plant uses was influenced by the culture of people which included ethnic group, language, acquired practical experiences [14]. As an example, in the NorthEast and Central-East of Côte d'Ivoire, four Agni tribes used wild edible plants in two different vegetation. Agni-Barabo and Agni-Bini shared their knowledge [51]. In fact, they shared the same migration histories, the same religion and the same vegetation $[14-39,49]$. Meanwhile, the intercultural variation on the common antimalarial plant species, was not significantly different, in this study. Indeed, the common plant species usually shared, are actively used through time and are considered effective. For example, in the South-Central of Ethiopia, four ethnic communities living in two different areas (Gurage, Mareqo, Qebena and Silti), have used the common plants and on the same time are considered effective $[13,48]$. In contrary, two ethnic groups Koulango and Lobi, living around the East side of the Comoé National Park of Côte d'Ivoire, were compared about the availability of wild edible plants [52]. These authors have shown in their studies that, in spite of their close contact, Koulango and Lobi ethnic groups like differently 
wild edible plants. In fact, their studies have revealed that the uses of a given wild plant species are specific to one ethnic group and its culture $[12,39]$. Otherwise, the knowledge sharing depends on the ethnic groups [13], and the preference of the plant species uses [12]. For example, 14 different ethnic groups in the Northern of Benin, were compared through the uses of the parts of Parkia biglobosa (Jacq.) G.Don [53]. In their studies, local knowledge varied from one ethnic group to another according to the form of uses and the organs of Parkia biglobosa, in the same geographical area. In fact, Lokpa, Waama and Bariba ethnic groups assigned high consensus value for bark, leaves, roots, seeds and pulp. In contrast, Nago, Anii, Dendi, Otamari, Mokolé, Foodo, Yom, Berba, Boko and assigned low value to bark and leaves. For the different form of uses of Parkia biglobosa, there is a consensus values for decoction, condiments were high for Bariba, Dendi, Fulani, Waama and Lokpa but low for other ethnic groups.

On the other hand, the extent of the knowledge on the medicinal plants according to the $\mathrm{FL}$, changes from one country to another. As an example, in the rural communities of Dhirkot, Azad Jammu and Kashmir in Pakistan, preferred different plants to cure specifically wound healing, gastrointestinal disorders, body weakness, diabetes and cough [3].

\section{Conclusion}

An ethnobotanical study on the perception of malaria and cultural diversity of antimalarial plants used by three sympatric ethnic groups, was conducted in the Department of Alépé, Southeast of Côte d'Ivoire. Six various forms of malaria were observed within the ethnic groups. A total of 77 antimalarial plants distributed in 71 genera and 38 families were collected. Our results show that there is a similar perception on five forms of malaria through all ethnic groups. Exceptionally, Akyé and Gwa ethnic groups perceive a mystical form of malaria. However, seven most important antimalarial plant species, were highlighted Annickia polycarpa (DC) Setten \& Maas, Gymnanthemum amygdalinum (Delile) Sch. Bip. ex Walp, Alstonia boonei De Wild. Nauclea latifolia Sm., Harungana madagascariensis Lam. ex Poir., Ocimum gratissimum L. and Senna occidentalis (L.) Link. Nevertheless, local people share 20 antimalarial plant species with a similar proximity within Akyé and Gwa ethnic groups. Even so, there is no specific plant species which cure a particular form of malaria.

In conclusion, traditional medicine plays a significant role in local people's daily life. In spite of their different migration histories, the close contact of these ethnic groups promote the sharing of the knowledge. In fact, people use the same important plants to cure malaria and know overall five forms of malaria. Exceptionally, Akyé and Gwa ethnic groups know both the sixth form, mystical malaria, due to their geographical proximity. Knowledge on antimalarial plants in study area does not reach a stable climax, but could be evolved by trial and error, as effective cures malaria.

\section{Declarations}

\section{Ethics approval and consent to participate}


The present study is purely based on a field survey instead of humans. Before starting investigation, the chief of each investigated village was informed on the research project. Then, an agreement was needed to residents prior to start questions following the recommendations of the International Society of Ethnobiology Code of ethics for the publication of this research and any accompanying images.

\section{Consent for publication}

Will be provided

\section{Availability of data and materials}

The authors declare that data supporting the findings of this study are available within the article. However, the raw files (Microsoft excel, World) can be provided by the authors on request.

\section{Competing interests}

The authors state that they have no competing interest.

\section{Funding}

This paper is a part of a master's thesis of A.L. DIOP (first author). However, no funding was provided by any source to conduct this survey.

\section{Author contributions}

All the authors contributed in a fundamental way to this work. D.F. MALAN and A.L. DIOP designed the subject and the methodological approach. DIOP led the field data collection and performed the data analysis with M.D. KOUGBO and DF MALAN. All the authors have critically read this article and approved it as the final manuscript.

\section{Acknowledgements}

Local inhabitants of the study area are gratefully acknowledged for sharing valuable information. Our sincere thanks go to Mrs Attokpa from the "English" teaching unit of the Nangui Abrogoua University who kindly read and correct the manuscript.

\section{References}


1. de Boer HJ, Lamxay V, Bjork L. Comparing medicinal plant knowledge using similarity: A case of the Brou, Saek and Kry in Lao PDR. J Ethnopharmacol. 2012; doi: 10.1016/j.jep.2012.03.017

2. Maroyi A. Traditional use of medicinal plants in southcentral Zimbabwe: review and perspectives. J Ethnobiol Ethnomed. 2013 ; doi: 10.1186/1746-4269-9-31.

3. Farooq A, Amjad MS, Ahmad K, Altaf M, Umair M, Abbasi AM. Ethnomedicinal knowledge of the rural communities of Dhirkot, Azad Jammu and Kashmir, Pakistan. J. Ethnobiol. Ethnomed. 2019; doi:10.1186/s1300201903232

4. Bla KB, Trebissou JND, Bidié ADP, Assi YJ, Zihiri-Guédé N, Djaman AJ. Étude ethnopharmacologique des plantes antipaludiques chez les BaoulésN'Gban Toumodi dans le Centre de la Côte d'Ivoire. J Appl Biosci. 2015; doi:10.4314/jab.v85i1.4

5. Kipré GR, Offoumou MR, Silué KD, Bouabré GM, Zirihi-Guédé N, Djaman AJ. Enquête ethnopharmacologique des plantes antipaludiques dans le Département d’Agboville, Sud-Est de la Côte d'Ivoire. J Appl Biosci. 2017; doi:10.4314/jab.v109i1.6

6. Diarra N, van't Klooster C, Togola A, Diallo D, Willcox M, de Jong J. Ethnobotanical study of plants used against malaria in Sélingué subdistrict, Mali. J Ethnopharmacol. 2015; doi:10.1016/j.jep.2015.02.054

7. Traoré MS, Baldé MA, Diallo MST, Baldé ES, Diané S, Camara A, Diallo A, Baldé A, Kéïta A, Kéita SM, Oularé K, Magassouba FB, Diakité I, Diallo A, Baldé AM. Ethnobotanical survey on medicinal plants used by Guinean traditional healers in the treatment of malaria. J Ethnopharmacol. 2013; doi:10.1016/j.jep.2013.10.048

8. Youmsi RDF, Fokou PVT, Menkem EZ, BakarngaVia I, Keumoe R, Nana V, Boyom FF. Ethnobotanical survey of medicinal plants used as insects repellents in six malaria endemic localities of Cameroon. J Ethnobiol Ethnomed. 2017; doi 10.1186/s130020170155x

9. Yetein MH, Houessou LG, Lougbégnon TO, Teka O, Tente B. Ethnobotanical study of medicinal plants used for the treatment of malaria in the plateau of Allada, Benin (West Africa). J Ethnopharmacol. 2013; doi:10.1016/j.jep.2012.12.022

10. Ngarivhume T, van't Klooster CIEA, Jong JTVM, Van der Westhuizen JH. Medicinal plants used by traditional healers for the treatment of malaria in the Chipinge District in Zimbabwe. J Ethnopharmacol. 2015; doi:10.1016/j.jep.2014.11.011

11. Kujawska M, Hilgert NI, Keller HA, Gil G. Medicinal plant diversity and diversity and intercultural interactions between indigenous Guarani, Criollos and Polish migrants in subtropics of Argentina. PLOS one. 2017; doi:10.1371/journal.pone.0169373

12. Vodouhê FG, Coulibaly O, Greene C, Sinsin B. Estimating the local value of non-timber forest products to Pendjari biosphere reserve dwellers in Benin. Eco Bot. 2009;doi.org/10.1007/s1223100991027

13. Teka A, Asfaw Z, Demissew S, Van Damme P. Medicinal plants use practice in four ethnic communities (Gurage, Mareqo, Qebena and Silti), south central Ethiopia. J Ethnobiol Ethnomed. 2020;doi:10.1186/s130020200033771 
14. Avocèvou-Ayisso C, Avohou TH, Oumorou M, Dossou G, Sinsin B. Ethnobotany of Pentadesma butyracea in Benin: a quantitative approach. Ethnobot Res Application 2012;10:151-166.

15. Bouquet A and Debray M. Plantes médicinales de Côte d'Ivoire. Paris, Mémoires ORSTOM ; 1974.

16. N’Guessan K, Tra Bi FH, Koné MW. Étude ethnopharmacologique de plantes antipaludiques utilisées en médecine traditionnelle chez les Abbey et Krobou d'Agboville (Côte d'Ivoire). Ethnopharmacol. 2009;44:42-50.

17. Malan DF, Neuba DFR, Kouakou KL. Medicinal plants and traditional healing practices in ehotile people, around the aby lagoon (eastern littoral of Côte d'Ivoire). J Ethnobiol Ethnomed. 2015; doi:10.1186/s1300201500048

18. Hilgert NI, Gil GE. Reproductive medicine in northwest Argentina: traditional and institutional systems. J Ethnobiol Ethnomed. 2007;doi: 10.1186/17464269319

19. Leonti M, Casu L, Sanna F, Bonsignore L. A comparison of medicinal plants use in Sardinia and Sicily De Materia Medica revisited. J Ethnopharmacol. 2009; doi: 10.1016/j.jep.2008.10.027

20. Kossonou KT, Assanvo AD. Linguistique et migration des peuples en Côte d'Ivoire: cas des Akan (Kwa). Revue du CAMES : Littérature, langues et linguistique. $2016 ; 4: 106-119$

21. Allou KR, Gonnin G. Côte d'Ivoire : Les premiers habitants. Abidjan, CERAP ; 2006. p. $29-97$.

22. Diabaté DH. La formation des sanvi dans le sanvi, un royaume Akan (17011901). Luballery, Paris, Nouvelle Imprimerie; 2013.

23. Adjélou JB. Société traditionnelle et économie monétaire en pays Akyé de 1875 à 1946. [PhD thesis]. [Abidjan, Côte d'Ivoire] Université Félix Houphouët Boigny, UFR Sciences de l'homme et de la Société; 2016.

24. Goly AM. Culture Ghwa: Histoire et Tradition. Abidjan, Graphic Excel ; 2010. p. 31169

25. Aka K. Traditions musicales chez les Akan lagunaires de Côte d'Ivoire : cas des Abbey, Abidji, Ehotilé et M'batto. Tervuren, Belgium: Musée royal de l'Afrique centrale. 2011.

26. WHO. Global Health Observatory (GHO) data, Côte d'Ivoire: country profiles. 2020. Available from: https://www.who.int/gho/countries/civ/country_profiles/en/. Accessed 30 Jul 2020.

27. Guillaumet JL, Adjanohoun E. Végétation de la Côte d’Ivoire. In: Avenard JM, Eldin M, Girard G, Sircoulon J, Touchebeu P, Perraud A, editors. Le milieu naturel de Côte d'Ivoire. Mémoires ORSTOM; 1971. p. 161-168.

28. Bamba S, Gonnin G, Kodjo GN, Nagnin O, Ouattara FT, Tiacoh C, Yao K, Zunon JG. Abidjan, Côte d’Ivoire. Mémorial de la Côte d'Ivoire; 1989

29. Quinlan M. Considerations for collecting free lists in the field: examples from ethnobotany. Field Methods 2005; 17:1-16

30. Sutrop U. List task and a cognitive salience index. Field methods. 2001;doi: $10.1177 / 1525822 \times 0101300303$

31. 31. Jaccard P. Nouvelles recherches sur la distribution florale. Bull Société Vaudoise Sci Nat 1908 ; 44 :223-270 
32. Friedman J, Yaniv Z, Dafni A, Palewitch D. A preliminary classification of the healing potential of medicinal plants, based on a rational analysis of an ethnopharmacological field survey among Bedouins in the Negev Desert, Israel. J. Ethnopharmacol. 1986;16:275-287.

33. Kffuri CW, Lopes MA, Ming LC, Odonne G, Kinupp VF. Antimalarial plants used by indigenous people of the Upper Rio Negro in Amazonas, Brazil. J Ethnopharmacol. 2016;doi: 0.1016/j.jep.2015.11.048

34. Dolo A, Maïga B, Dara V, Tapily A, Tolo Y, Arama C, Daou M, Doumbo O. Place of malaria among febrile illnesses in two ethnic tribes living in sympatry in Mali from 1998 to 2008. Bull. Soc. Pathol. Exot 2012; doi :10.1007/s1314901202496

35. Talapko J, Škrlec I, Alebić T, Jukić M and Včev A. Malaria: The Past and the Present Microorganisms. 2019 ; doi: 10.3390/microorganisms7060179

36. Gessler MC, Msuya DE, Nkunya MHH, Schär A, Heinrich M, Tanner M. Traditional healers in Tanzania: the perception of malaria and its causes. J Ethnopharmacol.

1995;doi:10.1016/03788741(95)01294N

37. Maffi L. Linguistic, Cultural and Biological diversity. Annu. Rev. Anthropol. 2005;doi: 10.1146/annurev.anthro.34.081804.120437

38. Menendez-Baceta G, Aceituno-Mata L, Reyes-Garcia V, Tardio J, Salpeteur M, Pardo-de-Santayana M. The importance of cultural factors in the distribution of medicinal plant knowledge: A case study in four Basque regions. J Ethnopharmacol. 2015;doi:10.1016/j.jep.2014.12.007

39. Gaoue OG, Coe MA, Bond M, Hart G, Seyler BC, McMillen H. Theories and major hypotheses in Ethnobotany. Econ Bot 2017;doi:10.1007/s1223101793898

40. Leonti M, Sticher O, Heinrich M. medicinal plants of the Popoluca, Mexico: Organoleptic properties as indigenous selection criteria. J Ethnopharmacol 2002;doi:10.1016/S03788741(02)000788

41. Atindehou KK, Schmid C, Brun R, Koné MW, Traoré D. Antitrypanosomal and antiplasmodial activity of medicinal plants from Côte d'Ivoire. J Ethnopharmacol 2007; doi:10.1016/j.jep.2003.09.032

42. Zirihi GN, Mambu L, Guédé-Guina F, Bodo B, Grellier P. In vitro antiplasmodial activity and cytotoxicity of 33 west African plants used for treatment of malaria. J Ethnopharmacol 2005; 98:281-285

43. Iwalena EO, Omisore ND, Adewunmi CO, Gbolade AA, Ademowo OG, Nneji C, Agboola OI, Daniyan OM. Antiprotozoan activities of Harungana madagascariensis stem bark extract on trichomonads and malaria. J Ethnopharmacol 2008;doi: 10.1016/j.jep.2008.02.019

44. Omoregie ES, Pal A, Sisodia B. In vitro antimalarial and cytotoxicity activities of leaf extracts of Vernonia amygdalina (Del). J Basic Appl Sci 2011;doi: 10.4314/njbas.v19i1.69356

45. Kayembe JS, Taba KM, Ntumba K, Kazadi TK. In vitro antimalarial activity of 11 terpenes isolated from Ocimum gratissimum and Cassia alata leaves. Screening of their binding affinity with Haemin. J plant stud 2012; doi: 10.5539/jps.v1n2p168

46. Tona L, Cimanga RK, Mesia K, Musuamba CT, De Bruyne T, Apers S, Hernans N, Van Miert S, Pieters L, Totté J, Vlietinck AJ. In vitro antiplasmodial activity of extracts and fractions from seven medicinal plants used in the Democratic Republic of Congo. J Ethnopharmacol 2005;doi:10.1016/j.jep.2004.02.022 
47. Okpekon T, Yolou S, Gleye C, Roblot F, Loiseau P, Bories C, Grellier P, Frappier F, Laurens A, Hocquemiller R. Anti-parasitic activities of medicinal plants used in Ivory Coast. J Ethnopharmacol. 2004;doi: 10.1016/j.jep.2003.09.029

48. Asase A, Hesse DE, Simmonds MSJ. Uses of multiple plants prescriptions for treatment of malaria by some communities in southern Ghana. J. Ethnopharmacol. 2012; doi:10.1016/j.jep.2012.09.028

49. Sylla Y, Silué KD, Ouattara K, Koné MW. Étude ethnobotanique des plantes utilisées contre le paludisme par les tradithérapeutes et herboristes dans le District d'Abidjan (Côte d'Ivoire). Int. J. Biol. Chem. Sci. 2018;doi:10.4314/ijbcs.v12i3.25

50. Leonti M, Casu L. Traditional medicines and globalization: current and future perspectives in ethnopharmacology. Front Pharmacol. 2013; doi: 10.3389/fphar.2013.00092

51. Malan DF, Litta AL, Kougbo MD, Diop AL, Kouassi KG. Wild edible plants in four Agni tribes of Centraleast and Northeast of Côte d'Ivoire: a comparative study. Biodiversitas 2020;doi:10.13057/biodiv/d211056

52. Kouakou YB, Malan DF, Kouassi KG, Diop AL, Bakayoko A. Disponibilité de quelques plantes alimentaires spontanées utilisées par les populations Koulango et Lobi de la périphérie est du parc National de la Comoé, Côte d'Ivoire. Afr Sci 2020; 16:33-50

53. Koura K, Ganglo JC, Assogbadjo AE, Agbangla C. Ethnic differences in use values and use patterns of Parkiabiglobosa in Northern Benin. J Ethnobiol Ethnomed 2011; 7:42.

\section{Figures}




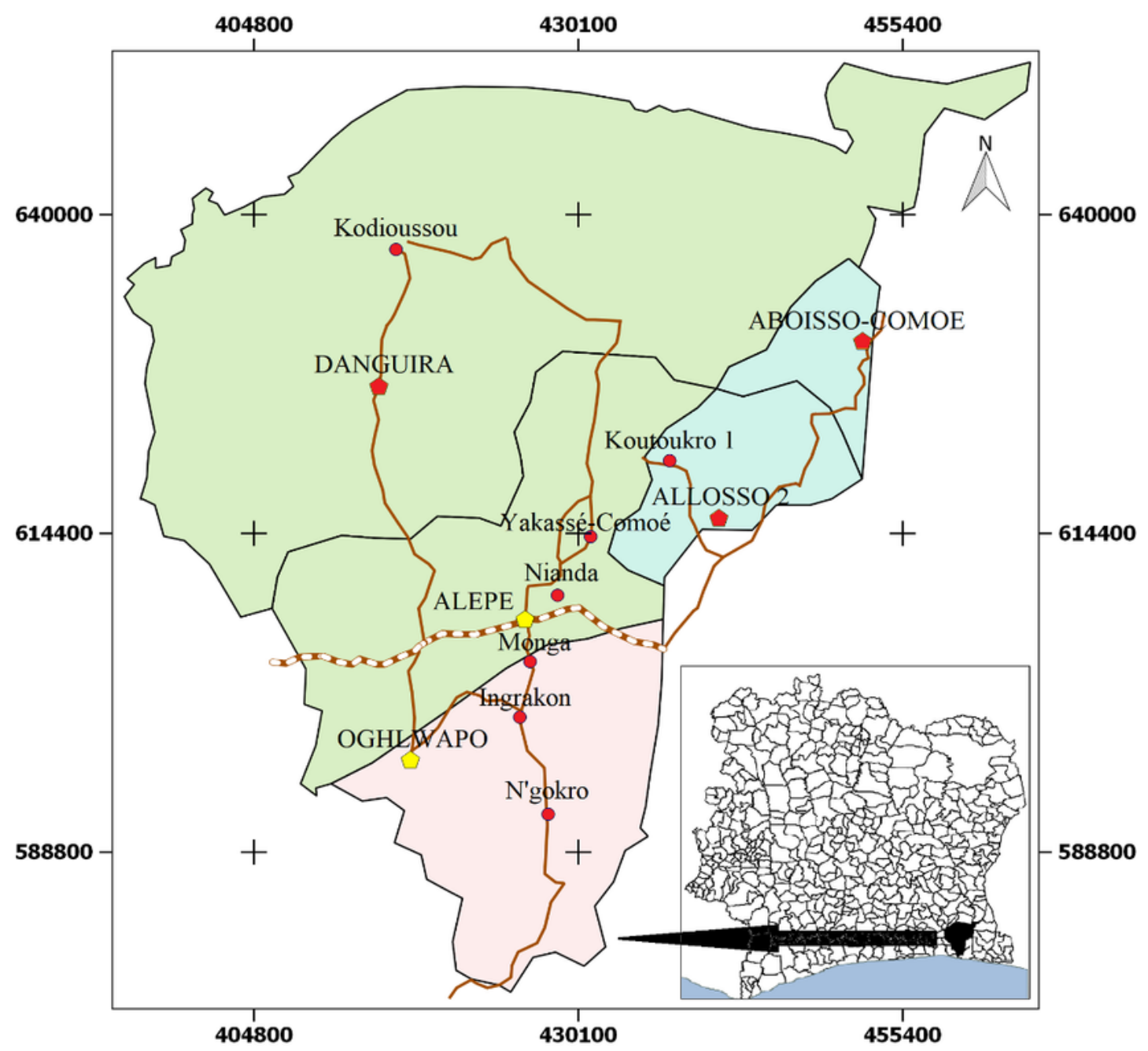

\section{LEGEND}

\footnotetext{
$\square$ Administrative centre of sub-prefecture

Surveyed administrative centre of sub-prefecture

- Surveyed village

Major road

- Main road
}

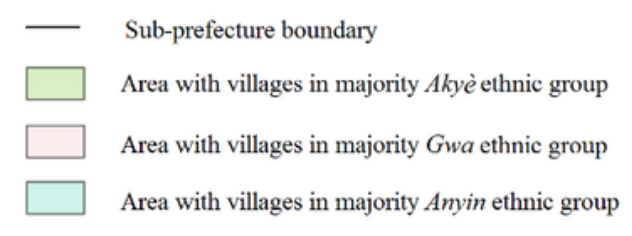

30 0

$30 \mathrm{~km}$

\section{Figure 1}

Location of Agni, Akyé and Gwa ethnic groups in Alépé Department. 


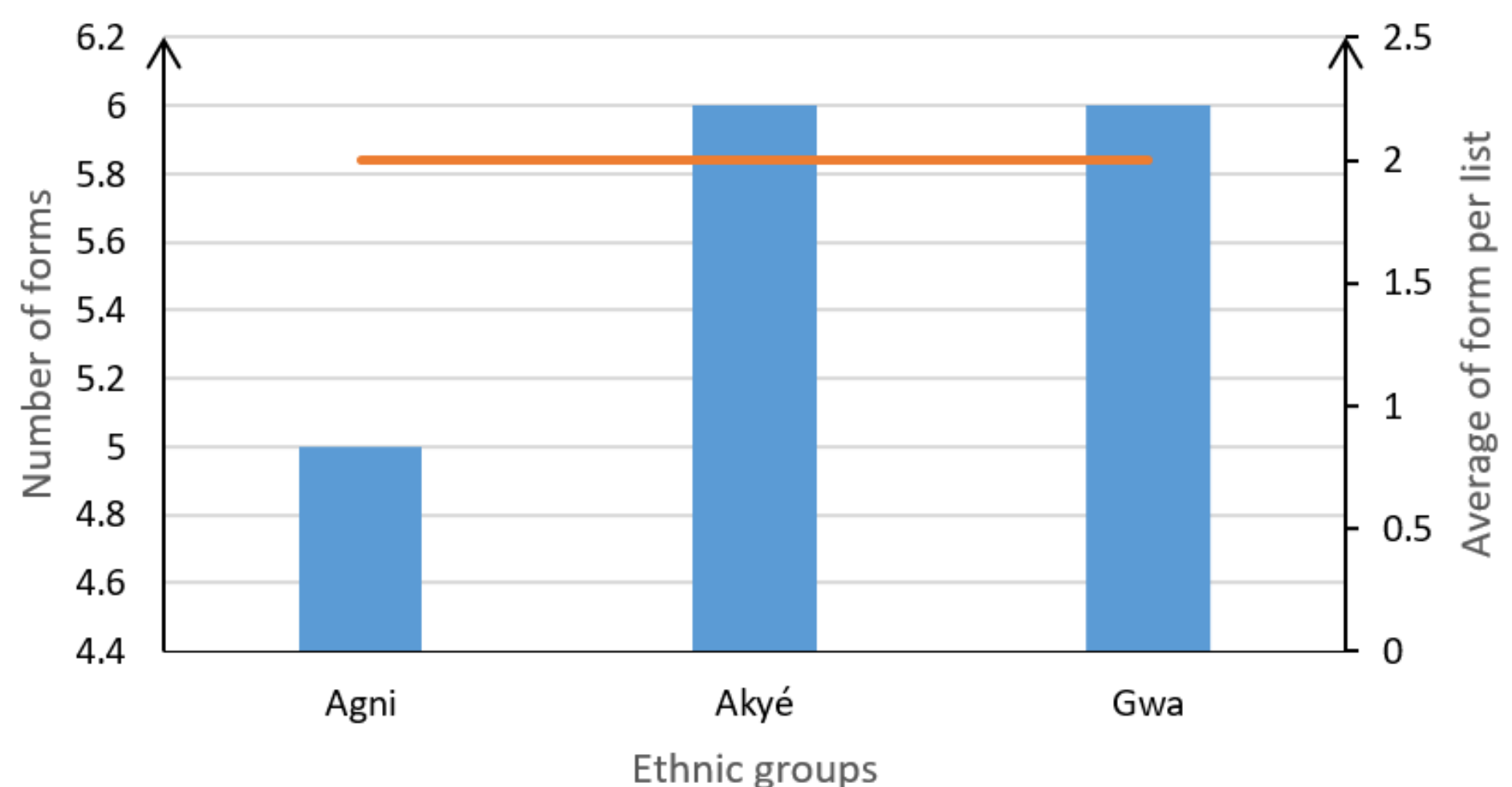

Figure 2

Forms of malaria mentioned by Agni, Akyé and Gwa ethnic groups. 


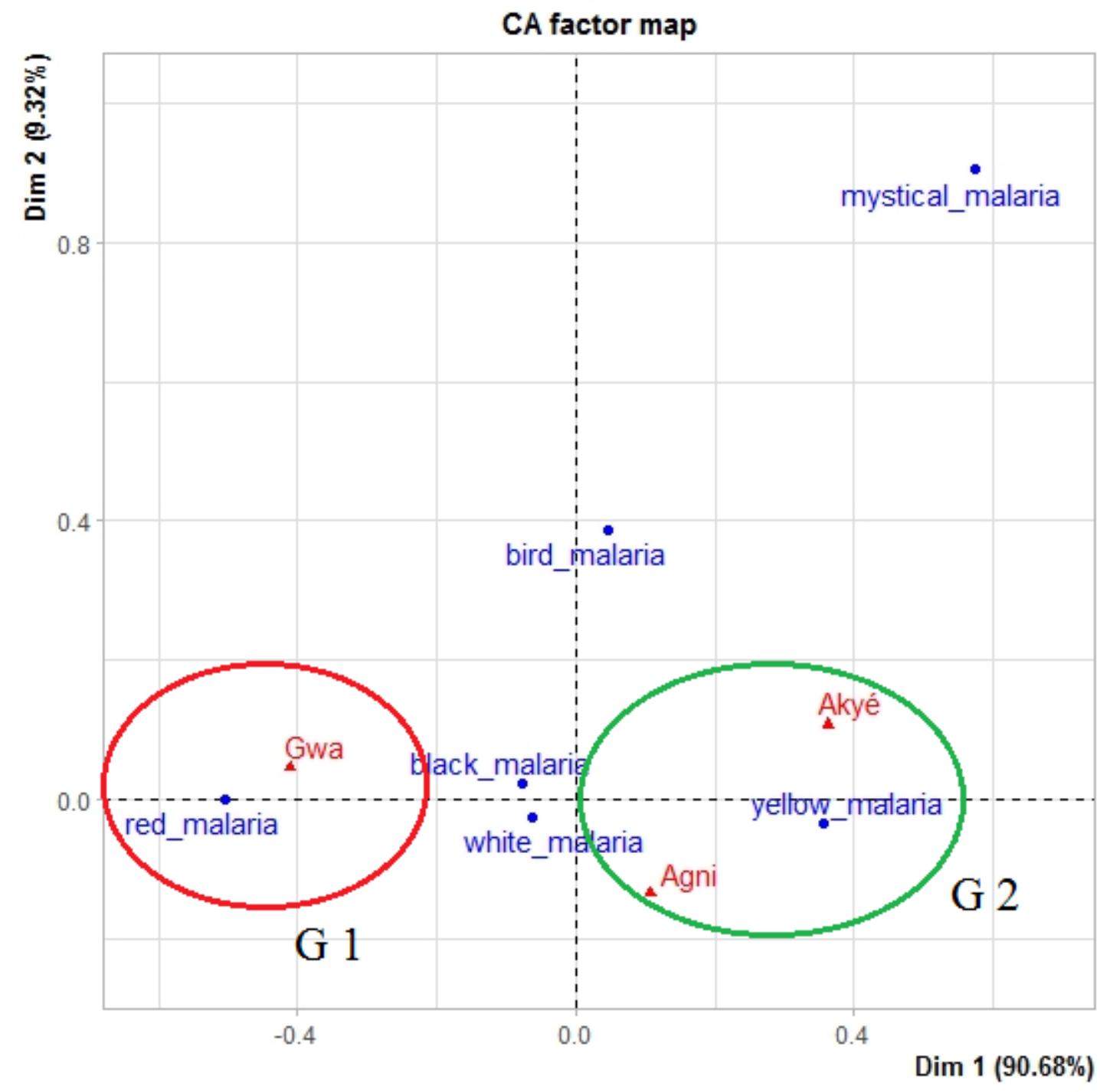

Figure 3

Distribution of the forms of malaria within the ethnic groups 


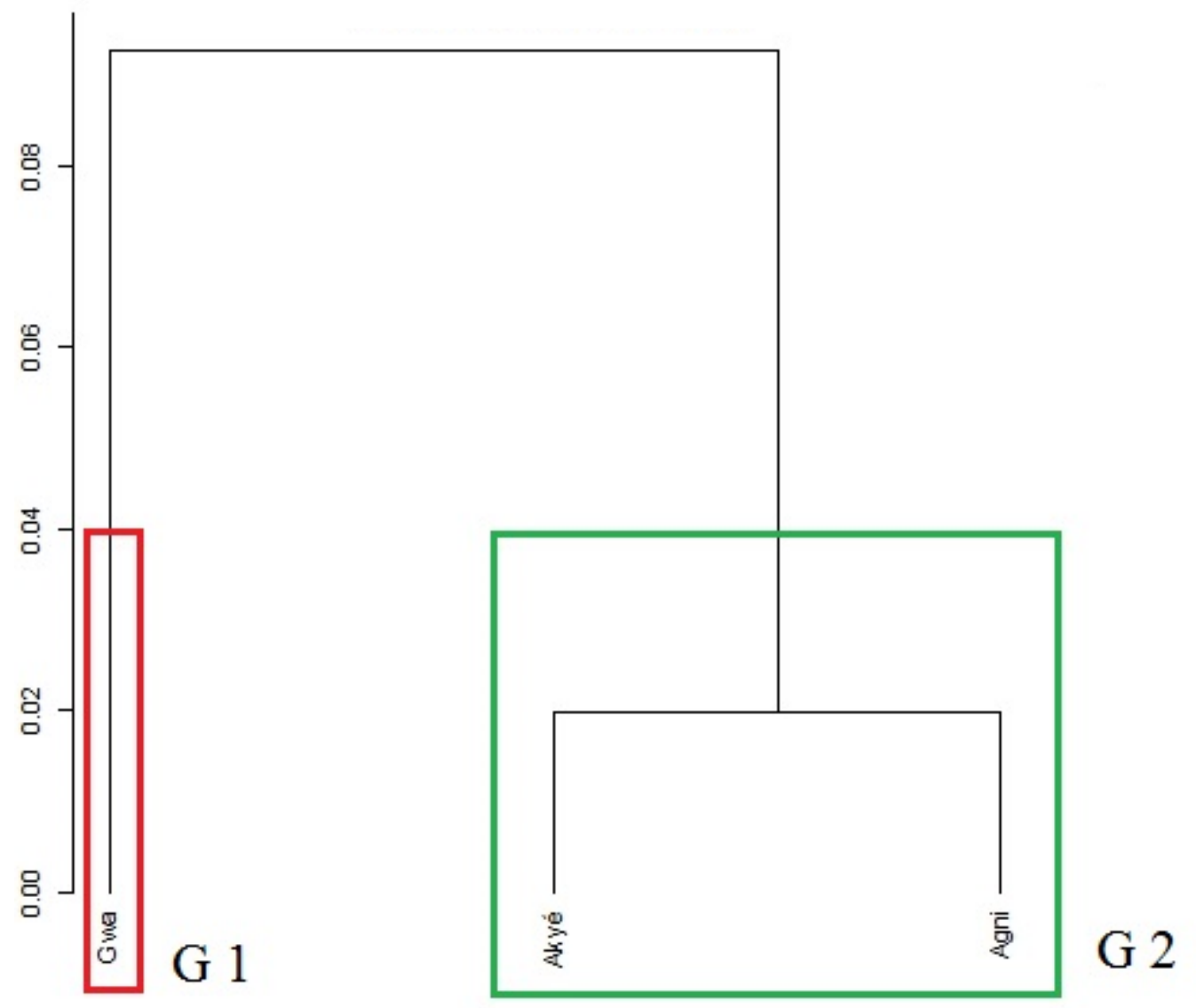

Figure 4

Hierarchical clusters of ethnic groups according to the forms of malaria. 


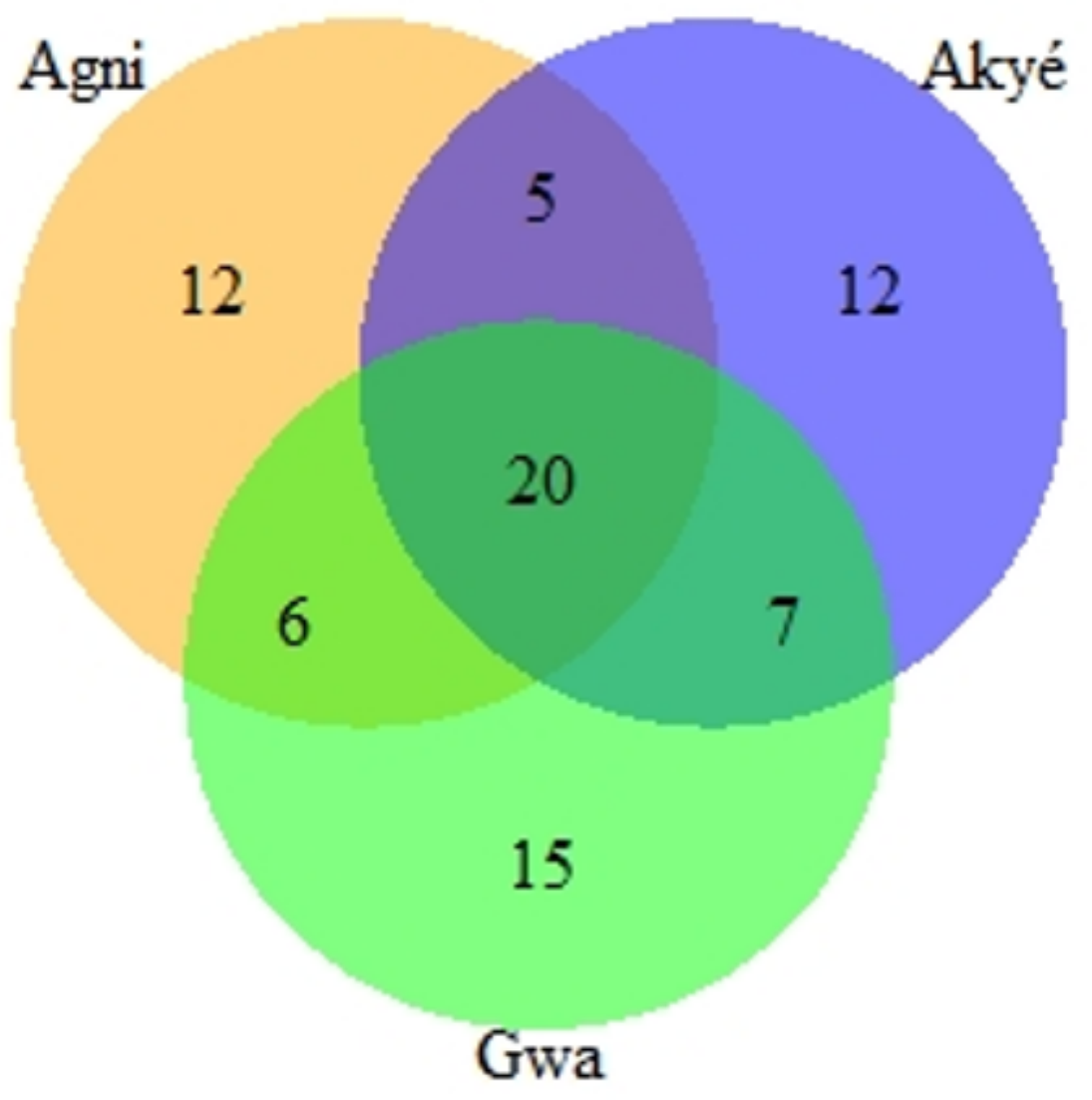

Figure 5

Venn diagram representing abundance of species used against malaria between ethnic groups of the study area. 

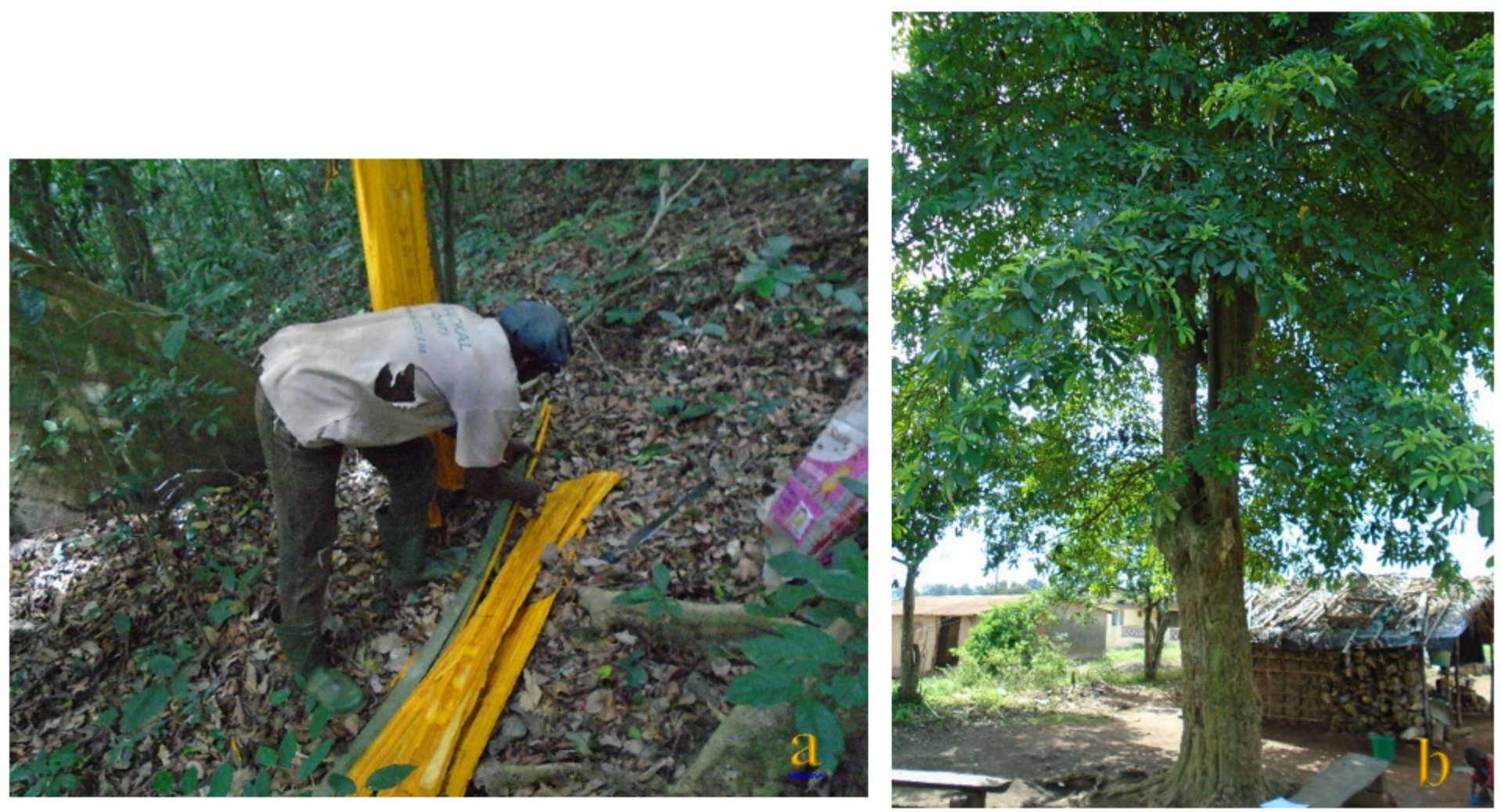

\section{Figure 6}

Two antimalarial plant species most important in the study area: a) bark pieces of Annickia polycarpa (DC.) Setten \& Maas collected by a key informant ; b) a tree of Alstonia boonei De Wild.

\section{A}

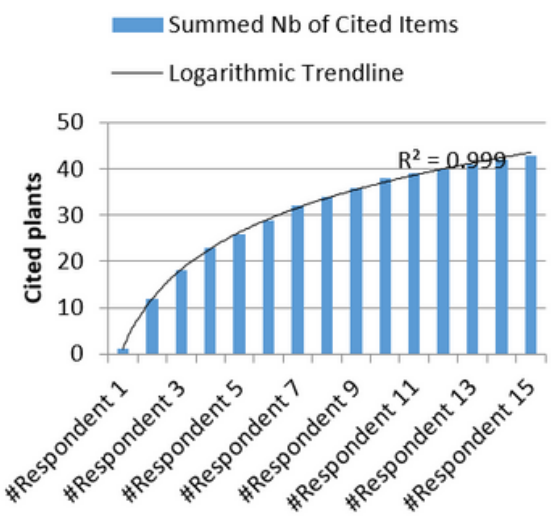

Summed $\mathrm{Nb}$ of Cited Items

—_ogarithmic Trendline

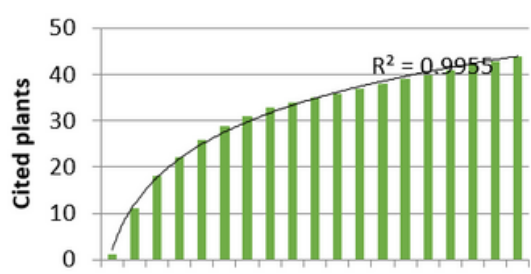

$x^{2} x^{3} x^{5} x^{1} x^{9}+x^{2}+x^{3}+x^{2}+x^{3}$

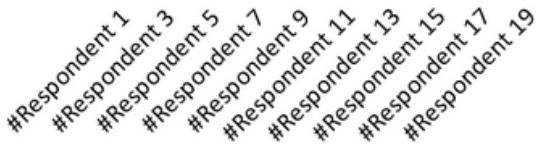

B
Summed Nb of Cited Items

- Logarithmic Trendline
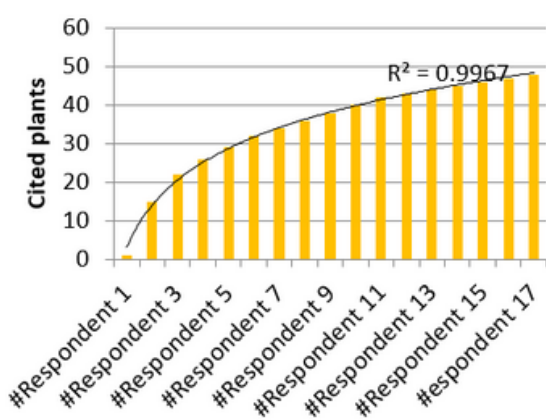

$\mathrm{C}$

Figure 7

Level of data saturation of antimalarial plants cited by: A) Agni; B) Akyé and C) Gwa people. 


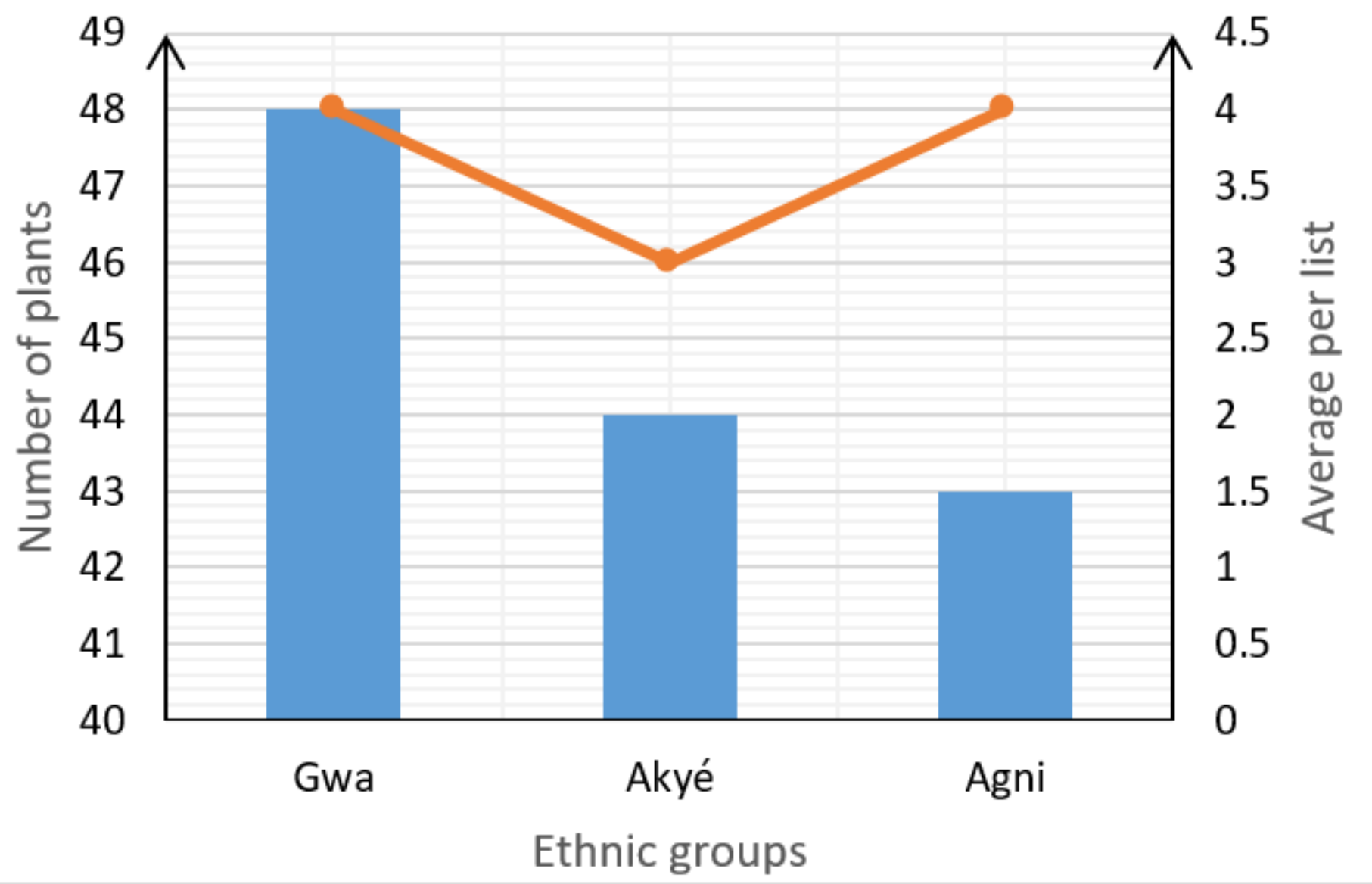

Figure 8

Number of antimalarial plants mentioned and average of plants per list in ethnic groups. 